\title{
Grassmann Electrodynamics and General Relativity
}

\author{
Denis Kochan \\ Department of Theoretical Physics, \\ Faculty of Mathematics Physics and Informatics, \\ Comenius University, \\ Mlynská Dolina F2, 842 48 Bratislava, Slovakia \\ e-mail: kochan@sophia.dtp.fmph.uniba.sk
}

\begin{abstract}
The aim of this paper is to present a short introduction to supergeometry on pure odd supermanifolds. (Pseudo)differential forms, Cartan calculus (DeRham differential, Lie derivative, "inner" product), metric, inner product, Killing's vector fields, Hodge star operator, integral forms, co-differential and connection on odd Riemannian supermanifolds are introduced. The electrodynamics and Einstein relativity with anti-commuting variables only are formulated modifying the geometry beyond classical (even, bosonic) theories appropriately. Extension of these ideas to general supermanifolds is straightforward.
\end{abstract}

PACS: 04.50. $+\mathrm{h}$; 04.20.Fy.

MSC: 58A10; 58A50; 32C81.

Key words: supergeometry; differential calculus; Grassmann electrodynamics.

\section{Introduction}

Supergeometry is an interesting and fruitful branch of mathematics with a variety of powerful applications in modern theoretical physics, in particular in SUSY, supergravity and superstrings. From a purely mathematical point of view, supergeometry is natural extension of the ordinary differential geometry by Grassmann variables. Such anti-commuting extensions represent an essential and inspiring feature of all supermathematics.

The first paper about supermathematics was the work of Martin [1], in which the classical limit of a system with fermionic degrees of freedom was discussed. This theory, later called pseudoclassical mechanics was independently developed in the middle of 70thies by Berezin and Marinov [2]-[3], Casalbuoni [4]-[5] and others. Since the Grassmann variables became an invaluable tool in the description of fermions, and because 
their natural combination with even (bosonic) degrees of freedom led at the beginning of 70thies to the discovery of supersymmetry, it was a necessary to build a rigorous mathematical theory, which would be able to describe both (even and odd) degrees of freedom. Systematical investigation in this direction was initiated at the beginning of 60thies by Berezin [6]-[7], but the main goals of the supermathematics were established during 70thies largely by the Russian mathematical school led by Berezin. More details about supergeometry (and also about its chronology) can be found in the review article [8], and in the famous Berezin book [9] which could be indeed regarded as the Bible of supermathematics (see also references therein).

The aim of this paper is to present a very short introduction to supergeometry over pure odd supermanifolds (sections 2-4). Using supergeometrical methods, we explain the electromagnetism described only by anti-commuting coordinates (section 5); such extravagant theory is called with a grain of salt Grassmann electrodynamics. After the definition of linear connection on pure odd Riemannian supermanifold we shall be able to reveal an odd analogy of the resulting theory with the Einstein theory of relativity (section 6). All this "odd business" (in both meanings of the word "odd") is based on classical geometrical analogy, similarly as pseudoclassical mechanics developed by Martin, Berezin and Casalbuoni.

\section{2 (Pseudo)differential forms}

The (pseudo)differential forms on an arbitrary smooth (real) $m \mid n$-dimensional supermanifold $\mathfrak{M}$ was in general investigated in the framework which is applied below in [10].

We shall study the basic properties of the (pseudo)differential forms on a pure odd (0|n-dimensional) real supermanifold, i.e. on $\mathbb{R}^{0 \mid n}$. The odd $n$-dimensional Cartesian space $\mathbb{R}^{0 \mid n}$ is covered by $n$ global Grassmann coordinates $\left(\xi^{1}, \ldots, \xi^{n}\right)$ and the superalgebra of functions $\mathcal{F}\left(\mathbb{R}^{0 \mid n}\right)$ coincides with exterior $\left(\mathbb{Z}_{2}\right.$-graded) algebra $\bigwedge \mathbb{R}^{n}=$ $\left[\bigwedge \mathbb{R}^{n}\right]_{[0]} \oplus\left[\bigwedge \mathbb{R}^{n}\right]_{[1]}$.

The tangent bundle of $\mathbb{R}^{0 \mid n}$ is a supermanifold $T \mathbb{R}^{0 \mid n}=\mathbb{R}^{0 \mid n} \times \mathbb{R}^{0 \mid n}$ with a set of global anti-commuting coordinates $\left(\xi^{1}, \ldots, \xi^{n}, \sigma^{1}, \ldots, \sigma^{n}\right)$ transforming under the transformation of coordinates $\xi^{\alpha} \mapsto \Xi^{\alpha}(\xi)$ on the base $\mathbb{R}^{0 \mid n}$ as

$$
\left(\xi^{\alpha}, \sigma^{\alpha}\right) \mapsto\left(\Xi^{\alpha}(\xi), \Sigma^{\alpha}(\xi, \sigma)=\sigma^{\beta} \frac{\partial \Xi^{\alpha}}{\partial \xi^{\beta}}\right)
$$

The odd functions $\Xi^{\alpha}(\xi)$ in (1) guarantee that the parity of coordinates on $T \mathbb{R}^{0 \mid n}$ is preserved. Throughout the paper, we use left derivatives with respect to Grassmann variables and Einstein summation convention. The parity of any object $O$ (with respect to anti-commuting variables) is denoted by $\tilde{O}$, and to distinguish Grassmann and ordinary variables we use Greek letters for the former and Latin letters for the latter. The odd tangent bundle $\Pi T \mathbb{R}^{0 \mid n}=\mathbb{R}^{0 \mid n} \times \mathbb{R}^{n}$ is a supermanifold which is obtained from $T \mathbb{R}^{0 \mid n}$ by changing the parity of the fiber variables $\sigma^{\alpha}$. The coordinate transformation 
on supermanifold $\mathbb{R}^{0 \mid n}$ induces the corresponding transformation on odd tangent bundle

$$
\left(\xi^{\alpha}, y^{\alpha}\right) \mapsto\left(\Xi^{\alpha}(\xi), Y^{\alpha}(\xi, y)=y^{\beta} \frac{\partial \Xi^{\alpha}}{\partial \xi^{\beta}}\right) .
$$

It is well known (for more details see [11], but we hope that it will become clear from our next explanation) that the superalgebra of differential forms on $\mathbb{R}^{0 \mid n}$ can be identified with $\mathbb{Z}_{2}$-graded algebra $\operatorname{Pol}\left(\Pi T \mathbb{R}^{0 \mid n}\right)=\operatorname{Pol}\left(\mathbb{R}^{n}\right) \otimes \bigwedge \mathbb{R}^{n}$ of all polynomials with real coefficients over supermanifold $\Pi T \mathbb{R}^{0 \mid n}$. There is one to one correspondence between the differentials of Grassmann variables $d \xi^{\alpha}$ (even quantities) and the even variables $y^{\alpha}$ covering fibers in $\Pi T \mathbb{R}^{0 \mid n}$. Their natural generalization leads to the definition of pseudodifferential forms over $\mathbb{R}^{0 \mid n}$, namely, the superalgebra of pseudodifferential forms is defined as the $\mathbb{Z}_{2}$-graded algebra $C^{\infty}\left(\Pi T \mathbb{R}^{0 \mid n}\right)=C^{\infty}\left(\mathbb{R}^{n}\right) \otimes \bigwedge \mathbb{R}^{n}$. The standard differential operations on forms, DeRham differential, Lie derivative and inner product, are identified with special vector fields on $\Pi T \mathbb{R}^{0 \mid n}$. To obtain their exact forms we use the fruitful idea of Maxim Kontsevich, who pointed out (see [12]) that the odd tangent bundle of arbitrary $m \mid n$-dimensional supermanifold $\mathfrak{M}$ is canonically isomorphic to the supermanifold of all supermaps $\mathbb{R}^{0 \mid 1} \rightarrow \mathfrak{M}$. In our case

$$
\Pi T \mathbb{R}^{0 \mid n} \equiv\left\{\text { supermaps: } \mathbb{R}^{0 \mid 1} \rightarrow \mathbb{R}^{0 \mid n}\right\} .
$$

An arbitrary supermap $\Phi \in \Pi T \mathbb{R}^{0 \mid n}$ is expressed in coordinates (by using Taylor expansion in $\theta$ ) as

$$
\Phi: \theta \mapsto \xi^{\alpha}(\Phi(\theta))=\xi^{\alpha}+\theta y^{\alpha} .
$$

It is clear that such $\Phi$ is characterized by $n$ odd and $n$ even coordinates, which transform in accordance with (2).

The supergroup Diff $\left(\mathbb{R}^{0 \mid 1}\right)=\left\{\right.$ diffeomorphisms: $\left.\mathbb{R}^{0 \mid 1} \rightarrow \mathbb{R}^{0 \mid 1} ; \theta \mapsto \theta^{\prime}=\theta a+\beta\right\}$ defines via its natural right action

$$
\Pi T \mathbb{R}^{0 \mid n} \times \operatorname{Diff}\left(\mathbb{R}^{0 \mid 1}\right) \rightarrow \Pi T \mathbb{R}^{0 \mid n}, \quad(\Phi, g) \mapsto \Phi \circ g,
$$

the left invariant (fundamental) vector fields $E, Q$ on $\Pi T \mathbb{R}^{0 \mid n}$. Their expression in coordinates is very simple, namely

$$
\begin{array}{ll}
E=y^{\alpha} \partial_{y^{\alpha}} & \text { Euler field }(\tilde{E}=0) \\
Q=y^{\alpha} \partial_{\xi^{\alpha}} & \text { DeRham differential }(\tilde{Q}=1) .
\end{array}
$$

The Euler vector field "measures" the degree of homogenity of (pseudo)differential forms under the supergroup action, therefore the superalgebra $C^{\infty}\left(\Pi T \mathbb{R}^{0 \mid n}\right)$ has also a natural $\mathbb{Z}$-graded structure $\left(f \in\left[C^{\infty}\left(\Pi T \mathbb{R}^{0 \mid n}\right)\right]^{(k)} \Leftrightarrow E f=k f=: \operatorname{deg}(f) f\right)$. A direct calculation gives the (super)commutation relations in the Lie superalgebra diff $\left(\mathbb{R}^{0 \mid 1}\right)$ :

$$
[E, E]=0, \quad[E, Q]=Q, \quad[Q, Q]=2 Q^{2}=0 .
$$


Similarly, the supergroup Diff $\left(\mathbb{R}^{0 \mid n}\right)=\left\{\right.$ diffeomorphisms of $\left.\mathbb{R}^{0 \mid n}\right\}$ acts on the odd tangent bundle $\Pi T \mathbb{R}^{0 \mid n}$,

$$
\operatorname{Diff}\left(\mathbb{R}^{0 \mid n}\right) \times \Pi T \mathbb{R}^{0 \mid n} \rightarrow \Pi T \mathbb{R}^{0 \mid n}, \quad(g, \Phi) \mapsto g \circ \Phi
$$

Therefore to any element $V=V\left(\xi^{\alpha}\right) \partial_{\xi^{\alpha}}=V^{\alpha}(\xi) \partial_{\xi^{\alpha}}$ of the corresponding Lie superalgebra diff $\left(\mathbb{R}^{0 \mid n}\right)=\mathfrak{X}\left(\mathbb{R}^{0 \mid n}\right)=\operatorname{Der}\left(\mathcal{F}\left(\mathbb{R}^{0 \mid n}\right)\right)$ we can assign unique vector field $V^{\uparrow}$ on $\Pi T \mathbb{R}^{0 \mid n}$. A straightforward coordinate computation ${ }^{1}$ gives

$$
V^{\uparrow}=V\left(\xi^{\alpha}\right) \partial_{\xi^{\alpha}}+(-1)^{\tilde{V}} Q\left(V\left(\xi^{\alpha}\right)\right) \partial_{y^{\alpha}} \Rightarrow \tilde{V^{\uparrow}}=\tilde{V}
$$

Apart from this natural lifting construction, it is also possible to associate to any $V \in \mathfrak{X}\left(\mathbb{R}^{0 \mid n}\right)$ certain vector field $V_{\uparrow}$ on $\Pi T \mathbb{R}^{0 \mid n}$ such that $\tilde{V}_{\uparrow}=\tilde{V}+1$ and

$$
\left[V_{\uparrow}, Q\right]=V^{\uparrow} .
$$

Obviously, the coordinate expression for $V_{\uparrow}$ is

$$
V_{\uparrow}=V\left(\xi^{\alpha}\right) \partial_{y^{\alpha}}
$$

For any vector fields $V, W \in \mathfrak{X}\left(\mathbb{R}^{0 \mid n}\right)$ it is easy to confirm the validity of supercommutations relations

$$
\begin{array}{lll}
{\left[E, V^{\uparrow}\right]=0,} & & {\left[E, V_{\uparrow}\right]=-V_{\uparrow},} \\
{\left[V^{\uparrow}, Q\right]=0,} & & {\left[V^{\uparrow}, W^{\uparrow}\right]=[V, W]^{\uparrow},} \\
{\left[V_{\uparrow}, W_{\uparrow}\right]=0,} & & {\left[V^{\uparrow}, W_{\uparrow}\right]=[V, W]_{\uparrow} .}
\end{array}
$$

The vector field $V^{\uparrow}$ corresponds to the Lie derivative $\mathcal{L}_{V}$ (with respect to $V$ ) acting on forms, whereas $V_{\uparrow}$ represents the inner product $i_{V}$ (with $V$ ). Equation (8) is the famous Cartan formula.

An arbitrary (pseudo)differential form is a polynomial (function) on the supermanifold $\Pi T \mathbb{R}^{0 \mid n}$ and therefore it can be expressed in any local coordinates as

$$
f=f(\xi, y)=\sum_{\beta=0}^{n} \sum_{\alpha_{1}, \ldots, \alpha_{\beta}} f_{\alpha_{1}, \ldots, \alpha_{\beta}}(y) \wedge \xi^{\alpha_{1}} \wedge \ldots \wedge \xi^{\alpha_{\beta}}
$$

with ordinary real polynomials (functions) $f_{\alpha_{1}, \ldots, \alpha_{\beta}}(y)$, which are skew-symmetric in the indices $\alpha_{1}, \ldots, \alpha_{\beta}$.

The integral of the pseudodifferential form $f$ over $\mathbb{R}^{0 \mid n}$ is defined as Berezin integral (for more details see [9],[11]) of a function $f$ on $\Pi T \mathbb{R}^{0 \mid n}$ :

$$
\mathbf{I}[f]:=\int_{\wedge \mathbb{R}^{n}} \overline{\mathrm{d} \xi} \int_{\mathbb{R}^{n}} \overline{\mathrm{d} y} f(\xi, y) .
$$

\footnotetext{
${ }^{1}$ In the case of an odd vector field $(\tilde{V}=1)$ it is necessary to consider instead of flow the superflow (homomorphism of the supergroups $\mathbb{R}^{1 \mid 1}$ and $\operatorname{Diff}\left(\mathbb{R}^{0 \mid n}\right)$ ), whose infinitesimal $(\Delta t, \Delta \epsilon)$ action in the coordinates is: $\xi^{\alpha} \mapsto \xi^{\alpha}+\Delta \epsilon V\left(\xi^{\alpha}\right)+\frac{\Delta t}{2}[V, V]\left(\xi^{\alpha}\right), \Delta \epsilon$ is odd variable.
} 
It is clear that such integral is not well defined for all elements of the superalgebras Pol $\left(\Pi T \mathbb{R}^{0 \mid n}\right)$ and $C^{\infty}\left(\Pi T \mathbb{R}^{0 \mid n}\right)$, because the manifold $\mathbb{R}^{n}$ (the typical fiber in $\Pi T \mathbb{R}^{0 \mid n}$ ) is not compact. The berezinian of the transformation (2) is equal to unity, therefore the integral (12) is coordinate independent; moreover, the odd per-partes integration formula (the odd Stokes theorem) holds,

$$
\mathbf{I}[(Q f) \wedge g]=(-1)^{\tilde{f}+1} \mathbf{I}[f \wedge(Q g)] \quad \Leftrightarrow \quad \mathbf{I}[Q(f \wedge g)]=0
$$

An arbitrary supermap $\Phi: \mathbb{R}^{0 \mid n} \rightarrow \mathbb{R}^{0 \mid m}$ defines (in accordance with (2)) the supermap $\Phi^{\uparrow}: \Pi T \mathbb{R}^{0 \mid n} \rightarrow \Pi T \mathbb{R}^{0 \mid m}$. The corresponding superalgebra homomorphism $\Phi^{\uparrow^{*}}$ : $C^{\infty}\left(\Pi T \mathbb{R}^{0 \mid m}\right) \rightarrow C^{\infty}\left(\Pi T \mathbb{R}^{0 \mid n}\right)$ is the pull-back of the supermap $\Phi$ over (pseudo)differential forms. For the vector field $V \in \mathfrak{X}\left(\mathbb{R}^{0 \mid n}\right)$, which generates the (super)flow (diffeomorphism) on the supermanifold $\mathbb{R}^{0 \mid n}$, the general formula for the pull-back reads:

$$
f \mapsto f_{(t, \epsilon)} \equiv\left[\Phi_{(t, \epsilon)}\left(V^{\uparrow}\right)\right]^{*} f= \begin{cases}\exp \left\{t V^{\uparrow}\right\} f & \text { for } \tilde{V}=0, \\ \exp \left\{\epsilon V^{\uparrow}+\frac{t}{2}\left[V^{\uparrow}, V^{\uparrow}\right]\right\} f & \text { for } \tilde{V}=1 .\end{cases}
$$

\section{Metric and Killing's vector fields}

The metric may be introduced on an arbitrary $m \mid n$-dimensional smooth supermanifold $\mathfrak{M}$ (in particular, on an ordinary manifold $M$ ) as an even regular (non-degenerate) quadratic function in fiber variables on a tangent bundle $T \mathfrak{M}$. In our case $\mathfrak{M}=\mathbb{R}^{0 \mid n}$ and the metric has the form

$$
g=g(\xi, \sigma)=g_{\alpha \beta}(\xi) \wedge \sigma^{\alpha} \wedge \sigma^{\beta},
$$

where functions $g_{\alpha \beta}(\xi)=-g_{\beta \alpha}(\xi)$ are even elements of $\mathcal{F}\left(\mathbb{R}^{0 \mid n}\right)$ (roughly speaking, components of metric tensor in coordinates $\left.\left(\xi^{1}, \ldots, \xi^{n}\right)\right)$. The non-degeneracy condition reads

$$
|g|:=\operatorname{det}\left(g_{\alpha \beta}\right) \neq 0 \text {. }
$$

Let us emphasize that non-degeneracy of $g$ implies that the even skew-symmetric matrix $g_{\alpha \beta}$ is invertible. Consequently, $0 \mid n$-dimensional supermanifold $\mathbb{R}^{0 \mid n}$ can be Riemannian only if $n$ is even (this fact is strongly reminiscent of the situation in symplectic geometry), therefore our next analysis will be performed only for pure odd, even-dimensional supermanifolds $\mathbb{R}^{0 \mid 2 n}$.

An arbitrary vector field $V=V\left(\xi^{\alpha}\right) \partial_{\xi^{\alpha}} \in \mathfrak{X}\left(\mathbb{R}^{0 \mid 2 n}\right)$ could be vertically lifted from the base supermanifold $\mathbb{R}^{0 \mid 2 n}$ to the tangent bundle $T \mathbb{R}^{0 \mid 2 n}$ : the coordinate expression for vertically lifted vector field over the tangent bundle is very simple

$$
V_{\uparrow_{v e r}}=V\left(\xi^{\alpha}\right) \partial_{\sigma^{\alpha}} \Rightarrow \tilde{V}_{\uparrow_{v e r}}=\tilde{V} .
$$

The metric $g$ on the supermanifold $\mathbb{R}^{0 \mid 2 n}$ allows us to define the inner product of vector fields on $\mathbb{R}^{0 \mid 2 n}$ as follows

$$
(V, W)_{g}:=(-1)^{\tilde{W}+1} V_{\uparrow_{v e r}}\left[W_{\uparrow_{v e r}}\left(\frac{g}{2}\right)\right]=V\left(\xi^{\alpha}\right) \wedge g_{\alpha \beta}(\xi) \wedge W\left(\xi^{\beta}\right) .
$$


It is clear that for all vector fields $V, W, U \in \mathfrak{X}\left(\mathbb{R}^{0 \mid 2 n}\right)$ homogeneous with respect to parity and arbitrary function $f \in \mathcal{F}\left(\mathbb{R}^{0 \mid 2 n}\right)$ the following relations are valid

$$
\left.\begin{array}{rl}
(\widehat{V, W})_{g} & =\tilde{V}+\tilde{W}, \\
(V, W)_{g} & =-(-1)^{(\tilde{V}+1)(\tilde{W}+1)}(W, V)_{g} \quad \text { odd graded skew-symmetry }, \\
(V+f U, W)_{g} & =(V, W)_{g}+f(U, W)_{g} \\
(V, W+f U)_{g} & =(V, W)_{g}+(-1)^{\tilde{V} \tilde{f}} f(V, U)_{g}
\end{array}\right\} \text { graded } f \text {-linearity . }
$$

In the ordinary differential geometry, it is well known, that apart from the vertical lifting procedure there also exists a canonical horizontal lift of vector field from the base $\mathbb{R}^{0 \mid 2 n}$ to the tangent bundle $T \mathbb{R}^{0 \mid 2 n}$. An arbitrary even [odd] vector field $V=$ $V\left(\xi^{\alpha}\right) \partial_{\xi^{\alpha}} \in \mathfrak{X}\left(\mathbb{R}^{0 \mid 2 n}\right)$ induces an infinitesimal flow [superflow] on the base supermanifold $\mathbb{R}^{0 \mid 2 n}$. Such infinitesimal diffeomorphism of $\mathbb{R}^{0 \mid 2 n}$, in accordance with (1), generates the (super)flow on the tangent bundle $T \mathbb{R}^{0 \mid 2 n}$. Its generator is the vector field

$$
V^{\uparrow h o r}=V\left(\xi^{\alpha}\right) \partial_{\xi^{\alpha}}+\left[\sigma^{\beta} \partial_{\xi^{\beta}}\left(V\left(\xi^{\alpha}\right)\right)\right] \partial_{\sigma^{\alpha}} \Rightarrow \tilde{V}^{\uparrow h o r}=\tilde{V} .
$$

The origin of the horizontal lifted vector field $V^{\uparrow \text { hor }} \in \mathfrak{X}\left(T \mathbb{R}^{0 \mid 2 n}\right)$ is the same as the origin of the vector field $V^{\uparrow} \in \mathfrak{X}\left(\Pi T \mathbb{R}^{0 \mid 2 n}\right)$, which acts as Lie derivative on the algebra of (pseudo)differential forms.

The conformal Killing's vector fields on the Riemannian supermanifold $\mathbb{R}^{0 \mid 2 n}$ are solutions of the system of $n(2 n-1)$ algebraic equations

$$
\chi \wedge g=V^{\uparrow h o r}(g) \Leftrightarrow \chi \wedge g_{\alpha \beta}=V^{\mu}\left(g_{\alpha \beta}\right)_{, \mu}+(-1)^{\tilde{V}}\left[g_{\alpha \mu}\left(V^{\mu}\right)_{, \beta}-g_{\beta \mu}\left(V^{\mu}\right)_{, \alpha}\right],
$$

where $\chi \in \mathcal{F}\left(\mathbb{R}^{0 \mid 2 n}\right)$ is even conformal scaling function and $(f)_{, \mu}=\partial_{\xi^{\mu}}[f(\xi)]$. It is evident that the linear combination of two conformal Killing's vector fields is again the conformal Killing's vector field, and because

$$
[V, W]^{\uparrow \text { hor }}=\left[V^{\uparrow \text { hor }}, W^{\uparrow h o r}\right]
$$

the supercommutator of two conformal Killing's vector fields is a generator of conformal transformation of the supermanifold $\mathbb{R}^{0 \mid 2 n}$, too.

It is possible to show that the Lie superalgebra of pure Killing vector fields $(\chi=0)$ over $\mathbb{R}^{0 \mid 2 n}$ is at a most $n(2 n+1) \mid 2 n$-dimensional subsuperalgebra of $n 2^{(2 n)} \mid n 2^{(2 n)}$-dimensional $\mathbb{Z}_{2}$-graded algebra $\mathfrak{X}\left(\mathbb{R}^{0 \mid 2 n}\right)$. The proof is analogical as in the ordinary differential geometry, but we do not prove this statement here, because it is not necessary for our next construction and, moreover, it requires the definition of a new supergeometrical notion, namely, the exponential (super)mapping.

Let us note that in analogy with ordinary differential geometry it is possible in supergeometry to define objects, similarly as it was done with metric, which correspond (from ordinary geometrical point of view) to covariant [contravariant] symmetric and 
anti-symmetric tensors:

Covariant [contravariant] symmetric tensor field of rank $k$ over an arbitrary smooth supermanifold $\mathfrak{M}$ (in particular, on an ordinary manifold $M$ ) is defined as the polynomial function of degree $k$ in fiber variables on the tangent [cotangent] bundle TM $\left[T^{*} \mathfrak{M}\right\rceil$.

Anti-symmetric covariant [contravariant] tensors (differential forms [multivector fields]) are analogically encoded in the polynomials in fiber variables on the odd tangent [odd cotangent] bundle $\Pi T \mathfrak{M}\left[\Pi T^{*} \mathfrak{M}\right]$. More detailed (but not exhaustive) description of the tensorial supercalculus on smooth supermanifolds may be found, e.g. in [9],[11],[13].

\section{$4 \quad$ Hodge $*_{g, o}$, Integral forms and Co-differential}

As in the case of ordinary differential forms, the metric is an essential ingredient in the definition of Hodge $*_{g, o}$. Because now we are familiar with all its relevant ingredients, we are able to define this operator.

For the function $f=f(\xi, y) \in\left[C^{\infty}\left(\Pi T \mathbb{R}^{0 \mid 2 n}\right)\right]^{(k)}$ ((pseudo)differential forms over $\mathbb{R}^{0 \mid 2 n}$ ) the Hodge $*_{g, o}$ operator is formally defined by its Fourier transform in the fibre variables $y^{\alpha}$, namely

$$
\left(*_{g, o} f\right)(\xi, y):=\frac{(\imath)^{\hat{f}}}{(2 \pi)^{n}} \int_{\mathbb{R}^{2 n}} \overline{\mathrm{d} z}(o \sqrt{|g|}) \wedge f(\xi, z) \wedge \exp \left\{-\imath z^{\alpha} g_{\alpha \beta} y^{\beta}\right\}
$$

where the symbol $\hat{f}$ denotes the parity with respect to even variables, i.e $\hat{f}=k \mid \bmod 2$ and the orientation $o= \pm 1$ (because the square root of $|g|$ is uniquely defined up to sign). In what follows, to simplify notation, we will put $O=1$ and subscript $o$ will be omitted. It is clear that the Hodge $*_{g}$ operator is defined on elements from Pol $\left(\Pi T \mathbb{R}^{0 \mid 2 n}\right)$ only in sense of distributions. Such generalized functions with one point support on the supermanifold $\Pi T \mathbb{R}^{0 \mid 2 n}$ are called integral forms over the odd tangent bundle and we denote them as $*\left(\operatorname{Pol}\left(\Pi T \mathbb{R}^{0 \mid 2 n}\right)\right)$. A straightforward calculation shows that the definition of the Hodge star operator does not dependent on the choice of coordinates. The definition (22) is strictly correct only for functions from $C^{\infty}\left(\Pi T \mathbb{R}^{0 \mid 2 n}\right)$ that are behaving well in the variables $y^{\alpha}$ at infinity (e.g. functions with compact support). The basic properties of the Hodge star operator can be obtained from the definition (22):

$$
\widehat{*_{g} f}=\hat{f}, \quad \widetilde{*_{g} f}=\tilde{f}, \quad \widehat{Q\left(*_{g} f\right)}=\hat{f}+1, \quad \widehat{Q\left(*_{g} f\right)}=\tilde{f}+1, \quad *_{g}\left(*_{g} f\right)=(-1)^{\hat{f}} f .
$$

Similarly, like in the standard differential geometry, the metric $g$ and the DeRham differential $Q$ define a new operator $\delta_{g}$ acting on (pseudo)differential forms. This operator, called co-differential is defined by equation

$$
Q(h) \wedge *_{g} f-Q\left(h \wedge *_{g} f\right)=:(-1)^{\tilde{h}} h \wedge *_{g}\left(\delta_{g} f\right)
$$


where functions $h, f \in C^{\infty}\left(\Pi T \mathbb{R}^{0 \mid 2 n}\right)$ are homogenous elements with respect to Grassmann and fiber variables respectively. From this definition it follows

$$
\delta_{g}(f)=(-1)^{\hat{f}} *_{g} Q *_{g} f=\left[\frac{\partial}{\partial \xi^{\alpha}}-\frac{\partial \ln \sqrt{|g|}}{\partial \xi^{\alpha}}-y^{\mu} \frac{\partial g_{\mu \nu}}{\partial \xi^{\alpha}} g^{\nu \lambda} \frac{\partial}{\partial y^{\lambda}}\right] g^{\alpha \beta} \frac{\partial f}{\partial y^{\beta}}
$$

where $g^{\alpha \mu}(\xi)$ is inverse to the matrix $g_{\mu \beta}(\xi)$, i.e. $g^{\alpha \mu}(\xi) \wedge g_{\mu \beta}(\xi)=\delta_{\beta}^{\alpha}$. All basic properties of the co-differential on Riemannian supermanifold $\mathbb{R}^{0 \mid 2 n}$ are given by equation (24), and may be deduced from the properties of Hodge star operator and DeRham differential. It is immediately evident from (22) and (24) that for arbitrary (pseudo)differential form $f$ and any supermap $\Phi: \mathbb{R}^{0 \mid 2 n} \rightarrow \mathbb{R}^{0 \mid 2 m}$ it holds

$$
\Phi^{\uparrow *}\left[*_{g} f\right]=*_{\left(\Phi^{*} g\right)}\left(\Phi^{\uparrow *} f\right) \quad \text { and } \quad \Phi^{\uparrow *}\left[\delta_{g}(f)\right]=\delta_{\left(\Phi^{*} g\right)}\left(\Phi^{\uparrow *} f\right)
$$

where $\Phi^{*} g$ denotes the pull-back of the metric $g$ (function over the tangent bundle) with respect to supermap $\mathrm{d} \Phi$ (differential of the supermap $\Phi$ ).

The "inner product" of (pseudo)differential forms over $\mathbb{R}^{0 \mid 2 n}$ is a necessary tool for building a "reasonable" physical theory on such supermanifold. If $f, h$ are homogenous elements with respect to Grassmann variables from the superspace $\left[C^{\infty}\left(\Pi T \mathbb{R}^{0 \mid 2 n}\right)\right]^{(k)}$, then their "inner product" is defined by

$$
\langle f, h\rangle_{g}:=\mathbf{I}\left[f \wedge *_{g} h\right] .
$$

It is clear that the "inner product" $\langle., .\rangle_{g}$ is $\mathbb{R}$-linear and, moreover,

$$
\begin{aligned}
\langle f, h\rangle_{g} & =(-1)^{k}\langle h, f\rangle_{g}(-1)^{\tilde{f} \tilde{h}}, \\
0 & =\langle\text { Even in } \xi, \text { Odd in } \xi\rangle_{g}, \\
\langle Q(f), h\rangle_{g} & =(-1)^{\tilde{f}}\left\langle f, \delta_{g}(h)\right\rangle_{g} .
\end{aligned}
$$

Let us note that the Laplace-DeRham operator defined on (pseudo)differential forms by

$$
\Delta_{g}:=-\left(Q \delta_{g}+\delta_{g} Q\right)
$$

is self-adjoint with respect to the "inner product" (26). We use the name "inner product" in quotation marks ex industria, to emphasize the peculiar fact that $\langle., .\rangle_{g}$, given by (26), is not always non-degenerate (e.g. for the closed even 2-forms over supermanifold $\mathbb{R}^{0 \mid 2}$ with metric $g=\epsilon_{\alpha \beta} \wedge \sigma^{\alpha} \wedge \sigma^{\beta}$ it holds $\left.\langle., .\rangle_{g} \equiv 0\right)$.

\section{Grassmann Electrodynamics}

The symplectic mechanics and classical electrodynamics are undoubtedly nice and simple applications of differential geometry in classical physics. The Grassmann electrodynamics is a natural "grassmannisation" of the well known version of ordinary even electrodynamics to the odd one. The inspiration for such a bit extravagant theory is 
provided by Cartan calculus on a pure odd Riemannian supermanifold $\mathbb{R}^{0 \mid 2 n}$ and by the geometrical formulation of classical electrodynamics (see e.g., interesting monographs $[14],[15]$, or the textbook [16]).

The electromagnetic field on the supermanifold $\mathbb{R}^{0 \mid 2 n}$ ("Grassmann space-time") is described by the potential 1-form

$$
A=A(\xi, y)=\mathcal{A}_{\alpha}(\xi) y^{\alpha} \equiv \mathcal{A}_{\alpha}(\xi) \mathrm{d} \xi^{\alpha}, \text { where } \mathcal{A}_{\alpha}(\xi) \in\left[\bigwedge \mathbb{R}^{2 n}\right]_{[1]} .
$$

Since the space of all odd potential 1 -forms on the supermanifold $\mathbb{R}^{0 \mid 2 n}$ is finite dimensional, $\operatorname{dim}\left(\left[\operatorname{Pol}\left(\Pi T \mathbb{R}^{0 \mid 2 n}\right)\right]_{[1]}^{(1)}\right)=n 2^{2 n}$, the "Grassmann electromagnetism" possesses only finite number of degrees of freedom.

The closed electrodynamics 2-form

$$
F:=Q(A) \in\left[\operatorname{Pol}\left(\Pi T \mathbb{R}^{0 \mid 2 n}\right)\right]_{[0]}^{(2)},
$$

is invariant under the gauge transformations

$$
A \mapsto A^{\prime}=A+Q(f), \text { where } f \in\left[\bigwedge \mathbb{R}^{2 n}\right]_{[0]} \text {. }
$$

Externals sources in such theory are described by the current 1-from

$$
J=J(\xi, y)=\mathcal{J}_{\alpha}(\xi) y^{\alpha} \equiv \mathcal{J}_{\alpha}(\xi) \mathrm{d} \xi^{\alpha}, \text { where } \mathcal{J}_{\alpha}(\xi) \in\left[\bigwedge \mathbb{R}^{2 n}\right]_{[1]}
$$

The dynamics of the Grassmann electromagnetic field is governed by the action

$$
S[A, J, g]:=-\frac{1}{2}\langle Q(A), Q(A)\rangle_{g}+\langle J, A\rangle_{g},
$$

which is a functional (strictly speaking only a function, because the Grassmann electrodynamics has finite number of degrees of freedom) of the potential and current 1-forms $A$ and $J$ and also of the metric tensor $g$. Variation of the action (33) with respect to the potential $A$ (without any boundary condition) leads (in the case when $\langle., .\rangle_{g}$ is regular "inner product" over 1-forms) to the set of algebraic Grassmann-Maxwell equations, which can be written in a compact form as

$$
\delta_{g} F=-J .
$$

The Grassmann-Maxwell equations are internally consistent only if the current 1-form $J$ is co-closed, i.e. if

$$
\delta_{g} J=0 \Leftrightarrow Q\left(*_{g} J\right)=0
$$

This is the continuity equation for the external currents.

To bring this extravagancy to the top of its bent, we split coordinates covering "Grassmann space-time" $\mathbb{R}^{0 \mid 2 n}=\mathbb{R}^{0 \mid(2 n-1)} \oplus \mathbb{R}^{0 \mid 1}$ to the "space" coordinates $\left(\xi^{1}, \ldots, \xi^{2 n-1}\right)$ and the "time" coordinate $\left(\xi^{2 n}=\tau\right)$. We restrict the closed integral form $*_{g} J \in$ 
$*\left(\operatorname{Pol}\left(\Pi T \mathbb{R}^{0 \mid 2 n}\right)\right)$ to the "space" hyper-surface $\mathbb{R}^{0 \mid(2 n-1)}$. The Grassmann charge related to external sources is then defined as an integral of the restricted form $\left.\left(*_{g} J\right)\right|_{\substack{\xi^{2 n}=\tau \\ y^{2 n}=0}}$ over "Grassmann space" subsupermanifold $\mathbb{R}^{0 \mid(2 n-1)}$, namely

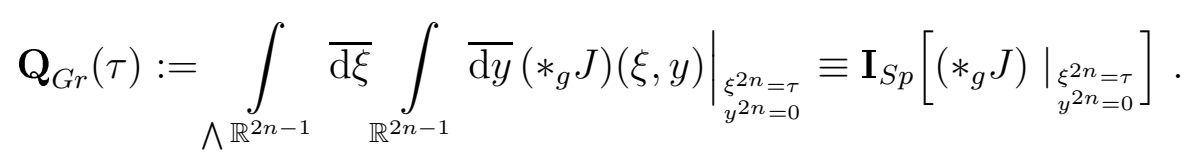

The odd vector field $\mathcal{T}=\partial_{\tau}$ defines a corresponding "Grassmann time" evolution. It is clear from equations (7), (8) and (35) that

$$
\mathcal{T}\left(*_{g} J\right)=\mathcal{T}^{\uparrow}\left(*_{g} J\right)=Q\left[\mathcal{T}_{\uparrow}\left(*_{g} J\right)\right]+\mathcal{T}_{\uparrow}\left[Q\left(*_{g} J\right)\right]=Q\left[\mathcal{T}_{\uparrow}\left(*_{g} J\right)\right] .
$$

Last equation and the odd Stokes theorem (13) imply that

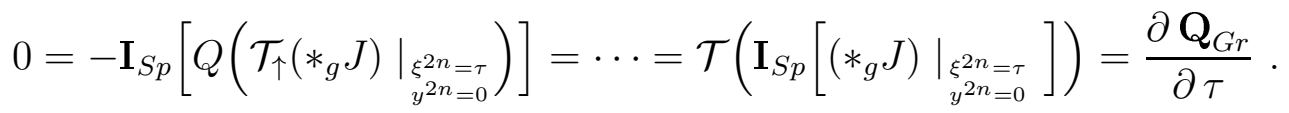

Let us remark that similarly as in the ordinary electromagnetism, it is possible to fix gauge freedom (of electromagnetic 2-form $F$ ) by imposing the condition

$$
\delta_{g} A=0 .
$$

This Lorentz gauge condition does not define the function $f$ in the gauge transformation (31) uniquely. Let us note that this uncertainty in the choice of the gauge fixing function $f$ is similar as in the ordinary electrodynamics: to any solution of the Poisson equation $\Delta_{g} f=\delta_{g} A$, which determines $f$, it is always possible to add a solution of the homogeneous (Laplace) equation.

To eliminate this ambiguity in the ordinary electromagnetism $\left(g_{\alpha \beta}=\eta_{\alpha \beta}\right.$, i.e. in $(3+1)$-Minkowski space-time), we need to fix the boundary conditions for the gauge fixing function $f$ (usually $f(x) \rightarrow 0$ for $x \rightarrow \infty$ ), because then the Poisson equation $\Delta_{\eta} f=\delta_{\eta} A$ has unique solution ${ }^{2}$.

Unfortunately, this does not work in the case of the Grassmann electrodynamics, where the boundary condition could be most naturally established by requirement that $0=$ $\left.f(\xi)\right|_{\xi=0}$ (the absolute term in $f$ does not contribute to gauge transformation (31)). The problem consists in an unpleasant fact that the Laplace-DeRham operator restricted to the functions ${ }^{3}$

$$
\Delta_{g} f=\delta_{g} Q(f)=\frac{\partial}{\partial \xi^{\alpha}} g^{\alpha \beta} \frac{\partial f}{\partial \xi^{\beta}}-\frac{\partial \ln \sqrt{|g|}}{\partial \xi^{\alpha}} g^{\alpha \beta} \frac{\partial f}{\partial \xi^{\beta}}
$$

is not invertible. This peculiar fact has a serious consequence: it is not possible to quantize the Grassmann-Maxwell theory by using Faddeev-Popov method in a straightforward way, because after applying gauge fixing condition (38) there still remains relative

\footnotetext{
${ }^{2}$ Let us note that this statement is not true for the general Riemannian manifold $(M, g)$, since the solution of the Poisson equation in particular physical situations constitutes a serious problem of mathematical physics.

${ }^{3}$ sometimes called as the Laplace-Beltrami operator
} 
"rich" gauge freedom, which is represented by the subgroup $\operatorname{Ker} \Delta_{g}$. Similar problems arise also with another gauge fixing conditions, e.g. $\xi^{\mu} \mathcal{A}_{\mu}(\xi)=0$ (radial gauge) or $\mathcal{A}_{2 n}(\xi)=0$ (Hamiltonian gauge) and therefore the problem of the quantization will not be discussed in this paper. The reason is down-to-earth, needs an individual approach from the case to case.

The Grassmann-Maxwell equations (34) could be rewritten in Lorentz gauge (38) in the form

$$
\Delta_{g} A=J .
$$

Now, let us consider the case of a free electromagnetic field $(J \equiv 0)$. The variation of the action (33) with respect to the metric $g$

$$
S[A, g+\Delta t h]=S[A, g]-\Delta t \int_{\wedge \mathbb{R}^{2 n}} \frac{\overline{\mathrm{d} \xi}}{\sqrt{|g|}} \frac{1}{2} h_{\alpha \beta}(\xi) T^{\alpha \beta}(\xi)+o(\Delta t),
$$

where $\Delta t h$ is the perturbation of metric ( $\Delta t$ is an infinitesimal real parameter) and $\overline{\mathrm{d} \xi} \sqrt{\left|g^{-1}\right|}$ is invariant Berezin integral measure, defines the covariant tensor field (a quadratic function in the fiber variables of the tangent bundle):

$$
T=T(\xi, \sigma)=T_{\alpha \beta}(\xi) \wedge \sigma^{\alpha} \wedge \sigma^{\beta}, \quad \text { where } T_{\alpha \beta}:=g_{\alpha \mu} g_{\beta \nu} T^{\mu \nu}
$$

It is the energy-momentum tensor of the free Grassmann electromagnetic field. It is clear from definition equation (41) that in the energy-momentum tensor $T$ only the skew-symmetric part is uniquely fixed (contrary to standard electrodynamics, where the same is true of the symmetric part). Namely,

$$
T_{\alpha \beta}=-\frac{1}{2} \int_{\mathbb{R}^{2 n}} \int_{\mathbb{R}^{2 n}} \frac{\overline{\mathrm{d} y \mathrm{~d} z}}{(2 \pi)^{n}}|g| \wedge\left\{g_{\alpha \beta}+\imath\left(z_{\alpha} y_{\beta}-z_{\beta} y_{\alpha}\right)\right\} \wedge\{F(\xi, y) \wedge F(\xi, z)\} \wedge e^{-\imath z^{\mu} g_{\mu \nu} y^{\nu}}
$$

where $z_{\alpha}=g_{\alpha \mu} z^{\mu}$ and $y_{\beta}=g_{\beta \nu} y^{\nu}$.

The energy-momentum tensor is a fundamental physical object that enables us to construct preserving quantities, which correspond to the space-time symmetries associated with (conformal) Killing's vector fields. Our next steps will be more general and final results could be automatically applied to Grassmann electrodynamics discussed above. Consider now an arbitrary field $\psi$ (function, 1-form, tensor field,...) over the supermanifold $\mathbb{R}^{0 \mid 2 n}$ with fixed metric $g$. Let us assume that the dynamics of the field $\psi$ is governed by the action

$$
S[\psi, g]=\int_{\wedge \mathbb{R}^{2 n}} \frac{\overline{\mathrm{d} \xi}}{\sqrt{|g|}}[L(\psi, g)](\xi)
$$

which is natural with respect to diffeomorphisms, i.e.

$$
\Phi^{*}(L(\psi, g))=L\left(\Phi^{*}(\psi), \Phi^{*}(g)\right)
$$


for any $\Phi \in \operatorname{Diff}\left(\mathbb{R}^{0 \mid 2 n}\right)$. If $V \in \mathfrak{X}\left(\mathbb{R}^{0 \mid 2 n}\right)$ is an arbitrary even vector field, $V$ defines the infinitesimal flow $\Phi_{\Delta t}(V)$ on the Riemannian supermanifold $\mathbb{R}^{0 \mid 2 n}$. The associated horizontally lifted even vector fields $V^{\uparrow}, V^{\uparrow}{ }^{\text {hor }}, \ldots$ generate the corresponding flows $\Phi_{\Delta t}\left(V^{\uparrow \ldots}\right)$ over supermanifolds $\Pi T \mathbb{R}^{0 \mid 2 n}, T \mathbb{R}^{0 \mid 2 n}, \ldots$ and consequently the pull-backs $\Phi_{\Delta t}^{*}\left(V^{\uparrow \ldots}\right)$ over forms, symmetric covariant tensors fields and so on. Since the berezinians of all such lifted flow-transformations are identically equal to unity and because the action (43) is natural with respect to supergroup Diff $\left(\mathbb{R}^{0 \mid 2 n}\right)$, we could write

$$
\begin{aligned}
S[\psi, g] & =S\left[\Phi_{\Delta t}^{*}\left(V^{\uparrow \ldots}\right)(\psi), \Phi_{\Delta t}^{*}\left(V^{\uparrow h o r}\right)(g)\right] \\
& =S\left[\psi+\Delta t V^{\uparrow \ldots}(\psi)+o(\Delta t), g+\Delta t V^{\uparrow h o r}(g)+o(\Delta t)\right] .
\end{aligned}
$$

If the field $\psi$ is the solution of the dynamical equations, i.e. $\frac{\delta S}{\delta \psi}=0$, previous equation implies that

$$
0=\int_{\wedge \mathbb{R}^{2 n}} \frac{\overline{\mathrm{d} \xi}}{\sqrt{|g|}} \frac{1}{2}\left(V^{\uparrow \text { hor }} g\right)_{\alpha \beta} T^{\alpha \beta}, \quad \text { where } \quad T^{\alpha \beta}(\xi)=-2 \sqrt{|g|} \frac{\delta S[\psi, g]}{\delta g_{\alpha \beta}(\xi)} .
$$

Explicit coordinate expression (20) for the horizontal lift $V^{\uparrow}$ hor ( $V$ is even) allows us to write

$$
\frac{1}{2}\left(V^{\uparrow \text { hor }} g\right)_{\alpha \beta} T^{\alpha \beta}=V^{\mu}\left[\frac{1}{2} \frac{\left(g_{\alpha \beta}\right)_{, \mu} T^{\alpha \beta}}{\sqrt{|g|}}-\left(\frac{g_{\mu \alpha} T^{\alpha \beta}}{\sqrt{|g|}}\right)_{, \beta}\right]-\left(\frac{V^{\mu} g_{\mu \alpha} T^{\alpha \beta}}{\sqrt{|g|}}\right)_{, \beta} .
$$

Since the equation (45) is valid for all even vector fields $V \in \mathfrak{X}\left(\mathbb{R}^{0 \mid 2 n}\right)$ (components $V^{\mu}(\xi)=V\left(\xi^{\mu}\right)$ are odd functions and $\left.\int \overline{\mathrm{d} \xi}()_{, \beta} \equiv 0\right)$, we conclude that

$$
\psi \text { satisfies } \frac{\delta S}{\delta \psi}=0 \Rightarrow \frac{1}{2} \frac{\left(g_{\alpha \beta}\right)_{, \mu} T^{\alpha \beta}}{\sqrt{|g|}}-\left(\frac{g_{\mu \alpha} T^{\alpha \beta}}{\sqrt{|g|}}\right)_{, \beta} \equiv 0 .
$$

This equation is a compact formulation of conservation laws, because from ordinary point of view, equation (47) is equivalent to the statement that $\left(T^{\mu \beta}\right)_{; \beta}=0$. Now, it becomes clear that for an arbitrary Killing's vector field $V \in \mathfrak{X}\left(\mathbb{R}^{0 \mid 2 n}\right)$ (the generator of an isometry of $\mathbb{R}^{0 \mid 2 n}$ ) the 1 -form

$$
T_{V}=T_{V}(\xi, y):=\left.\frac{1}{2} V_{\uparrow_{v e r}}\left(T_{\alpha \beta}(\xi) \wedge \sigma^{\alpha} \wedge \sigma^{b}\right)\right|_{\sigma \mapsto y}
$$

is co-closed, i.e. $Q\left(*_{g} T_{V}\right)=0$ (occasionally we are using metric to raise and lower indices, in order to make our final formulae more compact). If moreover $T_{\mu}^{\mu}=0$, the same is true for the arbitrary conformal Killing's vector field and therefore the quantity

$$
\mathbf{Q}_{V}(\tau):=\mathbf{I}_{S p}\left[\left.\left(*_{g} T_{V}\right)\right|_{\substack{\xi^{2 n}=\tau \\ y^{2 n}=0}}\right]
$$

is an integral of motion (i.e. $\partial_{\tau}\left[\mathbf{Q}_{V}(\tau)\right]=0$ ) and $T_{V}$ is corresponding Noether's current associated to the (conformal) Killing's vector field $V \in \mathfrak{X}\left(\mathbb{R}^{0 \mid 2 n}\right)$. The proof of this assertion is the same as for the Grassmann charge $\mathbf{Q}_{G r}$ discussed above. 


\section{Connection and Grassmann General Relativity}

In this section we shall try to uncover the Grassmann analogy of the Einstein's theory of General Relativity. We shall derive equations, which will govern the dynamics of the metric on the pure odd Riemannian supermanifold $\left(\mathbb{R}^{0 \mid 2 n}, g\right)$, but before this we are forced to establish a necessary supermathematical tool, namely the linear connection. The inspiration for such "monstrosity" comes from the standard General Relativity (see e.g., [17], [18]) and from the supergeometry discussed above.

We start with the definition of the linear connection (the quintessence of general relativity) on functions and vector fields for the general (not necessary Riemannian) pure odd supermanifolds. Connection (covariant derivative $\nabla$ with respect to vector field $V \in \mathfrak{X}\left(\mathbb{R}^{0 \mid n}\right)$ ) over functions $\mathcal{F}\left(\mathbb{R}^{0 \mid n}\right)$ is trivial: we require (analogically as in the ordinary case) that $\nabla_{V} f:=V f$. Connection over vector fields is the operation

$$
\nabla:\left(\mathfrak{X}\left(\mathbb{R}^{0 \mid n}\right)\right)^{2} \rightarrow \mathfrak{X}\left(\mathbb{R}^{0 \mid n}\right), \quad(V, W) \mapsto \nabla_{V} W,
$$

which in addition for arbitrary vector fields $V, W, U \in \mathfrak{X}\left(\mathbb{R}^{0 \mid n}\right)$ and function $f \in$ $\mathcal{F}\left(\mathbb{R}^{0 \mid n}\right.$ ) (all this objects are considered here and below as homogenous elements with respect to parity) satisfies:

$$
\begin{aligned}
\widetilde{\nabla_{V} W} & =\tilde{V}+\tilde{W} \\
\nabla_{V+f U} W & =\nabla_{V} W+f \nabla_{U} W \\
\nabla_{V}(W+f U) & =\nabla_{V} W+(V f) U+(-1)^{\tilde{V} \tilde{f}} f \nabla_{V} U .
\end{aligned}
$$

It is clear that the full information about connection $\nabla$ on supermanifold $\mathbb{R}^{0 \mid n}$ is uniquely encoded into $n^{3}$ Christoffel symbols (odd functions) $\Gamma_{\alpha \beta}^{\gamma}:=\left(\nabla_{\partial_{\xi^{\beta}}} \partial_{\xi^{\alpha}}\right)\left(\xi^{\gamma}\right)$, i.e. $\Gamma_{\alpha \beta}^{\gamma}(\xi)$ are components of the vector field $\nabla_{\partial_{\xi^{\beta}}} \partial_{\xi^{\alpha}}$ with respect to coordinates frame $\left(\xi^{1}, \ldots, \xi^{n}\right)$. If $\xi^{\alpha} \mapsto \Xi^{\alpha}(\xi)$, then $\Gamma^{\prime}$ 's transform as

$$
\Gamma_{\alpha \beta}^{\prime \gamma}=\frac{\partial \Xi^{\gamma}}{\partial \xi^{\lambda}} \frac{\partial \xi^{\mu}}{\partial \Xi^{\alpha}} \frac{\partial \xi^{\nu}}{\partial \Xi^{\beta}} \Gamma_{\mu \nu}^{\lambda}+\frac{\partial \xi^{\mu}}{\partial \Xi^{\alpha}} \frac{\partial \xi^{\nu}}{\partial \Xi^{\beta}} \frac{\partial}{\partial \xi^{\mu}}\left(\frac{\partial \Xi^{\gamma}}{\partial \xi^{\nu}}\right) .
$$

In the same spirit as in the case of ordinary connection, the torsion associated to connection $\nabla$ is the (super)tensorial (i.e. graded skew-symmetric and graded $f$-multilinear) operation $T_{\nabla}$ defined by

$$
\begin{aligned}
T_{\nabla}:\left(\mathfrak{X}\left(\mathbb{R}^{0 \mid n}\right)\right)^{2} & \rightarrow \mathfrak{X}\left(\mathbb{R}^{0 \mid n}\right), \\
(V, W) & \mapsto T_{\nabla}(V, W):=\nabla_{V} W-(-1)^{\tilde{V} \tilde{W}} \nabla_{W} V-[V, W]
\end{aligned}
$$

Let us note that $T_{\nabla}\left(\partial_{\xi^{\alpha}}, \partial_{\xi^{\beta}}\right)=\left(\Gamma_{\alpha \beta}^{\gamma}+\Gamma_{\beta \alpha}^{\gamma}\right) \partial_{\xi^{\gamma}}=T_{\nabla}\left(\partial_{\xi^{\beta}}, \partial_{\xi^{\alpha}}\right)$ and therefore the torsion vanishes identically only in the case, when the connection $\nabla$ on the supermanifold $\mathbb{R}^{0 \mid n}$ is anti-symmetric, i.e. $\Gamma_{\alpha \beta}^{\gamma}=-\Gamma_{\beta \alpha}^{\gamma}$. 
The curvature tensor $R_{\nabla}$ assigned to the connection $\nabla$ is naturally defined as the supermap

$$
\begin{aligned}
R_{\nabla}:\left(\mathfrak{X}\left(\mathbb{R}^{0 \mid n}\right)\right)^{3} & \rightarrow \mathfrak{X}\left(\mathbb{R}^{0 \mid n}\right), \\
(V, W, U) & \mapsto R_{\nabla}(V, W, U):=\left[\nabla_{V}, \nabla_{W}\right] U-\nabla_{[V, W]} U,
\end{aligned}
$$

where $\left[\nabla_{V}, \nabla_{W}\right]=\nabla_{V} \nabla_{W}-(-1)^{\tilde{V} \tilde{W}} \nabla_{W} \nabla_{V}$. Obvious coordinate expression for the curvature tensor $R_{\nabla}$ is

$$
R_{\nabla}\left(\partial_{\xi^{\mu}}, \partial_{\xi^{\nu}}, \partial_{\xi^{\beta}}\right)=\mathcal{R}_{\beta \mu \nu}^{\alpha} \partial_{\xi^{\alpha}}=\left\{\left(\Gamma_{\beta \mu}^{\alpha}\right)_{, \nu}+\left(\Gamma_{\beta \nu}^{\alpha}\right)_{, \mu}-\Gamma_{\beta \mu}^{\lambda} \Gamma_{\lambda \nu}^{\alpha}-\Gamma_{\beta \nu}^{\lambda} \Gamma_{\lambda \mu}^{\alpha}\right\} \partial_{\xi^{\alpha}} .
$$

Apart from the basic properties of the torsion $T_{\nabla}$ and curvature $R_{\nabla}$, which have arisen as straightforward consequences of their definitions, one also obtains two useful and powerful identities, namely the Ricci identity

$$
\sum_{\substack{\text { cycl. } \\ \text { permut. }}}(-1)^{\tilde{V} \tilde{U}}\left\{R_{\nabla}(V, W, U)-\nabla_{V}\left(T_{\nabla}(W, U)\right)-T_{\nabla}(V,[W, U])\right\}=0
$$

and the Bianchi identity

$$
\sum_{\substack{\text { cycl. } \\ \text { permut. }}}(-1)^{\tilde{V} \tilde{U}}\left\{\left[\nabla_{V}, R_{\nabla}(W, U, .)\right]+R_{\nabla}(V,[W, U], .)\right\}=0 .
$$

The proof of these assertions is not difficult, it is mainly a technical matter, which needs patience, large sheet of paper and knowledge about the (super)Jacobi identity stating that $(-1)^{\tilde{V} \tilde{U}}[V,[W, U]]+$ cycl. permut. $=0$ for any $V, W, U \in \mathfrak{X}\left(\mathbb{R}^{0 \mid n}\right)$.

On the odd Riemannian supermanifold $\left(\mathbb{R}^{0 \mid 2 n}, g\right)$ it is possible to require, analogically as in the ordinary case, the compatibility between connection $\nabla$ and metric $g$, which means that for arbitrary vector fields $V, W, U$ on $\mathbb{R}^{0 \mid 2 n}$ it should holds

$$
\nabla_{V}(W, U)_{g}=\left(\nabla_{V} W, U\right)_{g}+(-1)^{\tilde{V}(\tilde{W}+1)}\left(W, \nabla_{V} U\right)_{g} .
$$

As was mentioned above, the connection $\nabla$ can be uniquely defined by $(2 n)^{3}$-odd functions $(\Gamma)$, compatibility condition $(57)$ gives $2 n^{2}(2 n-1)$-secondary constrains on the Christoffel symbols

$$
\left(g_{\alpha \beta}\right)_{, \gamma}=g_{\alpha \mu} \Gamma_{\beta \gamma}^{\mu}-g_{\beta \mu} \Gamma_{\alpha \gamma}^{\mu} .
$$

But there still remains $2 n^{2}(2 n+1)$-degrees of freedom for the Christoffel $\Gamma$ 's, the residual ambiguity could be eliminated by requirement that connection $\nabla$ is without torsion $\left(T_{\nabla}=0\right)$ or equivalently that $\nabla$ is anti-symmetric. The final expression for the Christoffel symbols related to the metric and anti-symmetric connection $\nabla$ is very simple, and similar to the ordinary one. Namely,

$$
\Gamma_{\alpha \beta}^{\gamma}=\frac{1}{2} g^{\gamma \mu}\left[\left(g_{\mu \alpha}\right)_{, \beta}-\left(g_{\mu \beta}\right)_{, \alpha}-\left(g_{\alpha \beta}\right)_{, \mu}\right] \equiv g^{\gamma \mu} \Gamma_{\mu \alpha \beta} .
$$


The pure metricity of the connection $\nabla$ (in principle $T_{\nabla} \neq 0$ ) implies that

$$
\left(R_{\nabla}(V, W) X, Y\right)_{g}=(-1)^{(\tilde{X}+1)(\tilde{Y}+1)}\left(R_{\nabla}(V, W) Y, X\right)_{g},
$$

the last equation together with anti-symmetry of $\nabla$ reveal another interesting symmetry of the supermap $R_{\nabla}$ :

$$
\left(R_{\nabla}(V, W) X, Y\right)_{g}(-1)^{\tilde{Y}}=(-1)^{(\tilde{V}+\tilde{W})(\tilde{X}+\tilde{Y})}\left(R_{\nabla}(X, Y) V, W\right)_{g}(-1)^{\tilde{W}} .
$$

The proof of these identities is very simple and it is primarily based on the fact that for any function $f \in \mathcal{F}\left(\mathbb{R}^{0 \mid 2 n}\right)$ it holds $\left[\nabla_{V}, \nabla_{W}\right] f-\nabla_{[V, W]} f=0$. The choice $f=(X, Y)_{g}$ gives (59). The statement (60) is just a consequence of previous one together with Ricci identity without torsion terms.

Henceforth, we work only with the Grassmann-Levi-Civita $\nabla$, which is torsionless and compatible with metric $g$. In such case, it is evident that the curvature tensor $R_{\nabla}$ over the Riemannian supermanifold $\left(\mathbb{R}^{0 \mid 2 n}, g\right)$ has only $\frac{n^{2}}{3}\left[4 n^{2}-1\right]+n\left[4 n^{2}+1\right]$ independent components $\mathcal{R}_{\beta \mu \nu}^{\alpha}$, which is indeed more than in ordinary case for the same dimension.

Now we are able to describe the gravity on $\mathbb{R}^{0 \mid 2 n}$. The free dynamics of the metric $g$ is governed by the Grassmann-Hilbert action

$$
S_{G-H}[g]:=\int_{\wedge \mathbb{R}^{2 n}} \frac{\overline{\mathrm{d} \xi}}{\sqrt{|g|}} \mathcal{R},
$$

where the Ricci scalar $\mathcal{R}$ is defined by

$$
\mathcal{R}:=g^{\beta \alpha} \mathcal{R}_{\alpha \mu \beta}^{\mu}=g^{\beta \alpha}\left\{\left(\Gamma_{\alpha \beta}^{\mu}\right)_{, \mu}+\left(\Gamma_{\alpha \mu}^{\mu}\right)_{, \beta}-\Gamma_{\alpha \beta}^{\lambda} \Gamma_{\lambda \mu}^{\mu}-\Gamma_{\alpha \mu}^{\lambda} \Gamma_{\lambda \beta}^{\mu}\right\} .
$$

The first two terms in the action (61) can be simplified by per-partes integration $\left(\int \overline{\mathrm{d} \xi}()_{, \mu} \equiv 0\right)$. After such treatment we obtain more convenient expression, namely

$$
S_{G-H}[g]=\int_{\wedge \mathbb{R}^{2 n}} \frac{\overline{\mathrm{d} \xi}}{\sqrt{|g|}} \mathcal{G}, \quad \text { where } \mathcal{G}=g^{\beta \alpha}\left\{\Gamma_{\alpha \beta}^{\mu} \Gamma_{\mu \lambda}^{\lambda}+\Gamma_{\alpha \mu}^{\lambda} \Gamma_{\lambda \beta}^{\mu}\right\} .
$$

The variation of (63) with respect to the metric $g$ (without any boundary conditions, forasmuch as the surface terms do not contribute) leads to the system of algebraic equations

$$
\frac{\partial\left(\sqrt{\left|g^{-1}\right|} \mathcal{G}\right)}{\partial g_{\alpha \beta}}-\frac{\partial}{\partial \xi^{\mu}}\left[\frac{\partial\left(\sqrt{\left|g^{-1}\right|} \mathcal{G}\right)}{\partial\left(g_{\alpha \beta}\right)_{, \mu}}\right]=0
$$

which dictates the evolution of the free gravitational field. It is always possible to add to the Grassmann-Hilbert action a mater term of the form (43), and then the variation of $S_{G-H}[g]+S_{\text {mater }}[\psi, g]$ with respect to $g$, gives the Grassmann-Einstein equations

$$
\frac{\partial\left(\sqrt{\left|g^{-1}\right|} \mathcal{G}\right)}{\partial g_{\alpha \beta}}-\frac{\partial}{\partial \xi^{\mu}}\left[\frac{\partial\left(\sqrt{\left|g^{-1}\right|} \mathcal{G}\right)}{\partial\left(g_{\alpha \beta}\right)_{, \mu}}\right]=\frac{1}{2} \sqrt{\left|g^{-1}\right|} T^{\alpha \beta},
$$

where $T^{\alpha \beta}$ is the energy-momentum tensor (45) related with the mater fields. 


\section{Acknowledgement}

I would like to express my deep gratitude to my teacher P. Ševera, who was an excellent guide of mine to supergeometry. I am also very grateful to P. Prešnajder and V. Balek who gave me much valuable advice and who carefully read a draft of this paper and many thanks go to my girlfriend Tulka ( $)$ ) for her encouragement.

\section{References}

[1] J. L. Martin; Proc. Roy. Soc. A 251 (1959) 536.

[2] F. A.Berezin, M. S. Marinov; JETP Lett 21 (1975) 320.

[3] F. A.Berezin, M. S. Marinov; Ann. Phys. 104 (1977) 336.

[4] R. Casalbuoni; Nuovo Cimento A 33 (1976) 115 and 389.

[5] R. Casalbuoni; Phys. Lett B 62 (1976) 49.

[6] F. A. Berezin; Soviet Phys. Dokl. 6 (1961) 212.

[7] F. A. Berezin; The method of second quantization, Academic Press, New York, NY (1966).

[8] D. A. Leites; Russian Math. Surveys 35:1 (1980) 1.

[9] F. A. Berezin, A. A. Kirillov; Introduction to superanalysis, Reidel, Dordrecht (1987).

[10] D. Kochan; Pseudodifferential forms and supermechanics, submitted to Czech. Jour. of Phys.

[11] Yu. I. Manin; Gauge fields theory and complex geometry, Springer-Verlag, Berlin (1988).

[12] M. Kontsevich; Deformation quantization of Poisson manifolds, I, q-alg/9709040.

[13] B. S. DeWitt; Supermanifolds, University Press, Cambridge (1984).

[14] W. L. Burke; Applied differential geometry, University Press, Cambridge (1985).

[15] T. T. Frenkel; The geometry of physics, University Press, Cambridge (1998).

[16] M. Fecko; Differential geometry and Lie groups for physicists (in Slovak), to be published in 2003.

[17] S. Weinberg; Gravitation and cosmology, Wiley, New York (1972).

[18] L. D. Landau, E. M. Lifshitz; The classical theory of fields (4th ed.), Pergamon, London (1975). 